\title{
TOPOLOGY OF SOBOLEV MAPPINGS
}

\author{
Fengbo Hang and Fanghua Lin
}

\begin{abstract}
This paper addresses some topological and analytical issues concerning Sobolev mappings between compact Riemannian manifolds. Among the results we obtained are unified proofs of various generalizations of results obtained in a recent work of Brezis and Li. In particular we solved two conjectures in [BL]. We also give a topological obstruction for the weak sequential density of smooth maps in a given Sobolev mapping space. Finally we show a necessary and sufficient topological condition under which the smooth maps are strongly dense in the Sobolev spaces. The earlier result, Theorem 1 of [B2], was shown to be not correct.
\end{abstract}

\section{Introduction}

Here we give a brief exposition of some results shown in our recent work [HL]. Let $M$ be a compact smooth Riemannian manifold with or without boundary, and $N$ be a compact smooth Riemannian manifold without boundary. We shall always assume that $M$ and $N$ are connected and $N$ is isometrically embedded in $\mathbb{R}^{l}$ for some large $l$. For any $1 \leq p<\infty$, we consider the space of Sobolev mappings

$$
W^{1, p}(M, N)=\left\{u \in W^{1, p}\left(M, \mathbb{R}^{l}\right), u(x) \in N \text { a.e. }\right\}
$$

with $d(u, v)=\|u-v\|_{W^{1, p}\left(M, \mathbb{R}^{l}\right)}$ as the metric. From the calculus of variations, the following two mapping spaces are of interest,

$$
H_{S}^{1, p}(M, N)=\text { the strong closure of } C^{\infty}(M, N) \text { in } W^{1, p}(M, N),
$$

$H_{W}^{1, p}(M, N)=\left\{u \in W^{1, p}(M, N)\right.$ such that there exists a sequence $u_{j} \in$ $C^{\infty}(M, N)$ with $u_{j} \rightarrow u$ in $\left.W^{1, p}\left(M, \mathbb{R}^{l}\right)\right\}$. Obviously we have

$$
H_{S}^{1, p}(M, N) \subseteq H_{W}^{1, p}(M, N) \subseteq W^{1, p}(M, N) .
$$

Whether the above inclusions in (1.2) are strict or not is a difficult question and it has been studied by various authors. For the case $M=\mathbb{B}^{3}$ (a ball in $\mathbb{R}^{3}$ ), $N=\mathbb{S}^{2}$ and $p=2$, it was shown in $[\mathrm{BBC}]$ that $H_{W}^{1,2}\left(\mathbb{B}^{3}, \mathbb{S}^{2}\right)=W^{1,2}\left(\mathbb{B}^{3}, \mathbb{S}^{2}\right)$. On the other hand, it is rather easy to check $H_{S}^{1,2}\left(\mathbb{B}^{3}, \mathbb{S}^{2}\right) \neq W^{1,2}\left(\mathbb{B}^{2}, \mathbb{S}^{2}\right)$. In fact, in [B1], Bethuel gave some characterizations of maps in $H_{S}^{1,2}\left(\mathbb{B}^{3}, \mathbb{S}^{2}\right)$ (see also $[\mathrm{BCDH}]$ for some generalizations). Recently, Hardt and Rivière $[\mathrm{HR}]$ proved a necessary and sufficient condition for maps to lie in $H_{S}^{1,3}\left(\mathbb{B}^{4}, \mathbb{S}^{2}\right)$ in terms of certain mass of "minimal connections". For the general manifolds $M$

Received October 26, 2000. 
and $N$, some remarkable results were first established in [B2] (see $[\mathrm{H}]$ for an alternative approach of the main results of [B2] under some additional topological conditions). Recently some important progress was made in $[\mathrm{PR}]$ for sequentially weak closure of smooth maps and "minimal connections". We now turn to the question related to the topological information carried by maps in $W^{1, p}(M, N)$, $H_{S}^{1, p}(M, N)$ and $H_{W}^{1, p}(M, N)$ respectively. It was shown in [SU] that any map $f \in W^{1, p}(M, N)$ induces $f^{\#}: H^{k}(N, \mathbb{R}) \rightarrow H^{k}(M, \mathbb{R})$ for any $0 \leq k \leq[p]-$ 1 , a homomorphism between cohomology classes. We also note that Burstall [Bu] proved $W^{1,2}(M, N)$ maps induce conjugate classes of homomorphisms of $\pi_{1}(M) \rightarrow \pi_{1}(N)$, and Schoen-Yau [SY] showed that one can define conjugate classes of homomorphisms of $\pi_{n-1}(M) \rightarrow \pi_{n-1}(N)$ for $W^{1, n}(M, N)$ maps. The first systematic approach to these issues was due to White [W1] and [W2]. He proved, in particular, the following facts

(i) Let $d=\left\{\begin{array}{ll}{[p]} & \text { if } p \text { is not an integer } \\ p-1 & \text { if } p \text { is an integer }\end{array}\right.$, then each $f \in H_{W}^{1, p}(M, N)$ has a well-defined $d$ homotopy type which is preserved under sequentially weak convergence.

(ii) If $d=[p]-1$, then each $f \in W^{1, p}(M, N)$ has a well-defined $d$ homotopy type and it is preserved under sequentially weak convergence of maps.

(iii) Each map $f \in H_{S}^{1, p}(M, N)$ has a well-defined $[p]$ homotopy type that is preserved under the strong convergence of maps.

Recently, Brezis and $\mathrm{Li}[\mathrm{BL}]$ initiated the study of path connectedness of $W^{1, p}(M, N)$. Many results were proved and several open problems and conjectures were posed. Our present work is in fact motivated by these open problems and conjectures. To describe our main results, we first recall a few important notations. For convenience, let us assume $\partial M=\emptyset$ (the case $\partial M \neq \emptyset$ will be treated in Section 4 below).

Suppose $1 \leq p<\operatorname{dim} M$, we say two maps $u, v \in W^{1, p}(M, N)$ are $[p]-1$ homotopic, if for a generic $[p]-1$ skeleton $M^{[p]-1}$ of $M,\left.u\right|_{M[p]-1}$ and $\left.v\right|_{M[p]-1}$ are homotopic. Note that for generic $[p]-1$ skeletons, $u$ and $v$ are both $W^{1, p}$ on these skeletons and hence continuous. It, therefore, makes sense to say whether or not they are homotopic. It was proved in Section 3 of [W2] that this definition does not depend on the choices of the generic skeletons. We also recall (see [BL]) that for any $1 \leq p<\infty, u \sim_{p} v$ for $u, v \in W^{1, p}(M, N)$ if one may find a continuous path in $W^{1, p}(M, N)$ connecting $u$ and $v$. One of the main results of the present work is the following

Theorem 1.1. Let $M$ and $N$ be compact connected Riemannian manifolds without boundary, $1 \leq p<\operatorname{dim} M$, then for any $u, v \in W^{1, p}(M, N)$,

$$
u \sim_{p} v \Longleftrightarrow u \text { and } v \text { are }[p]-1 \text { homotopic. }
$$

With Theorem 1.1, one may reduce various questions concerning Sobolev mappings to purely topological ones. Indeed we have the following 
Proposition 1.1. Let $M$ and $N$ be compact connected Riemannian manifolds without boundary, $1 \leq p<\operatorname{dim} M$, for any smooth triangulation of $M$ the following two natural maps are bijections,

$$
C\left(M^{[p]}, N\right) / \sim_{M^{[p]-1}} \longleftarrow \operatorname{Lip}\left(M^{[p]}, N\right) / \sim_{M^{[p]-1}, \operatorname{Lip}} \longrightarrow W^{1, p}(M, N) / \sim_{p},
$$

here for $f, g \in C\left(M^{[p]}, N\right), f \sim_{M^{[p]-1}} g$ means $\left.f\right|_{M^{[p]-1}}$ and $\left.g\right|_{M^{[p]-1}}$ are homotopic, similarly, for $f, g \in \operatorname{Lip}\left(M^{[p]}, N\right), f \sim_{M^{[p]-1}, \text { Lip }} g$ means $\left.f\right|_{M^{[p]-1}}$ and $\left.g\right|_{M[p]-1}$ can be connected to each other by a continuous path in $\operatorname{Lip}\left(M^{[p]-1}, N\right)$. Also the natural map for left going arrow is the obvious one. The right going arrow is defined as follows, for any $f \in \operatorname{Lip}\left(M^{[p]}, N\right)$, let $u$ be the homogeneous degree zero extension of $f$ to higher dimensional simplices $M^{[p]+1}, \cdots, M^{\operatorname{dim} M}=$ $M$, then we send the equivalence class of $f$ to the equivalence class of $u$. This map is well defined by Theorem 1.1.

An immediate consequence of Proposition 1.1 is the following

Corollary 1.1. Let $M$ and $N$ be compact connected Riemannian manifolds without boundary, $1 \leq p<\operatorname{dim} M$, if $\pi_{i}(N)=0$ for $[p] \leq i \leq \operatorname{dim} M$, then the two maps below are bijections,

$$
C(M, N) / \sim_{M} \longleftarrow \operatorname{Lip}(M, N) / \sim_{M, L i p} \rightarrow W^{1, p}(M, N) / \sim_{p},
$$

the notations and maps are understood similarly as in Proposition 1.1.

We note that Theorem 6 of $[\mathrm{BL}]$ is contained in the above corollary. For the path connectedness of $W^{1, p}(M, N)$ we have

Proposition 1.2. Let $M$ and $N$ be compact connected Riemannian manifolds without boundary, $1 \leq p<\operatorname{dim} M$, if there exists a $k \in \mathbb{Z}, 0 \leq k \leq[p]-1$ such that $\pi_{i}(M)=0$ for $1 \leq i \leq k, \pi_{i}(N)=0$ for $k+1 \leq i \leq[p]-1$, then $W^{1, p}(M, N)$ is path connected.

We note Proposition 1.2 covers Theorem 2, Theorem 3 and Proposition 1 of [BL]. Recall that for any $p>q \geq 1$, we have a map $i_{p, q}: W^{1, p}(M, N) / \sim_{p} \rightarrow$ $W^{1, q}(M, N) / \sim_{q}$ defined in a natural way (see [BL]). Then we have a positive answer to the Conjecture 2 (and $2^{\prime}$ ) of $[\mathrm{BL}]$.

Proposition 1.3. Let $M$ and $N$ be compact connected Riemannian manifolds without boundary, $k \in \mathbb{Z}, k \geq 1, k \leq q<p<k+1$, then $i_{p, q}$ is a bijection.

We now turn to the question whether a given map $u \in W^{1, p}(M, N)$ can be connected to a smooth map by a continuous path in $W^{1, p}(M, N)$. It turns out that the question is closely related to whether such a map can be weakly approximated by a sequence of smooth maps in $W^{1, p}(M, N)$. Indeed we have the following two statements

Theorem 1.2. Let $M$ and $N$ be compact connected Riemannian manifolds without boundary, $1 \leq p<\operatorname{dim} M$. Given any $u \in W^{1, p}(M, N)$, then $u$ can be connected to a smooth map by a continuous path in $W^{1, p}(M, N)$ if and only if for 
any generic $[p]-1$ skeleton $M^{[p]-1},\left.u\right|_{M^{[p]-1}}$ has a continuous extension to $M$. The latter condition is also necessary for $u$ to be in $H_{W}^{1, p}(M, N)$.

Corollary 1.2. Let $M, N$ and $p$ be the same as in Theorem 1.2. Then every map in $W^{1, p}(M, N)$ can be connected to a smooth map if and only if for any smooth triangulation of $M$ (for definition of smoothness, see $p$. 77 of $[\mathrm{M}]$ ), any continuous map $f: M^{[p]} \rightarrow N$, we may find a continuous extension of $\left.f\right|_{M^{[p]-1}}$ to the whole $M$. The latter condition is also necessary for $H_{W}^{1, p}(M, N)$ to be equal to $W^{1, p}(M, N)$.

Naturally we conjecture that the topological condition in Theorem 1.2 is also sufficient for $u$ to be in $H_{W}^{1, p}(M, N)$ when $p \in \mathbb{Z}, 1 \leq p<\operatorname{dim} M$. Similarly we conjecture the topological condition in Corollary 1.2 is a sufficient condition for $H_{W}^{1, p}(M, N)$ to be equal to $W^{1, p}(M, N)$ when $p \in \mathbb{Z}, 1 \leq p<\operatorname{dim} M$. We refer to Section 3 below for examples that show such topological conditions in Theorem 1.2 and Corollary 1.2 may fail in general. Another direct consequence of Theorem 1.2 is the following

Corollary 1.3. Let $M, N$ and $p$ be the same as in Theorem 1.2. If either $[p]=1$ or $[p] \geq 2$ and $\pi_{i}(N)=0$ for $[p] \leq i \leq \operatorname{dim} M-1$, then any map in $W^{1, p}(M, N)$ can be connected by a continuous path in $W^{1, p}(M, N)$ to a smooth map.

We note Corollary 1.3 implies Theorem 5 of [BL]. Finally we come to the question of strong density of smooth maps in $W^{1, p}(M, N)$.

Theorem 1.3. Let $M$ and $N$ be compact connected smooth Riemannian manifolds without boundary, $1 \leq p<\operatorname{dim} M, u \in H_{S}^{1, p}(M, N)$, then when $p \notin \mathbb{Z}$, we have for any generic $[p]$ skeleton $M^{[p]},\left.u\right|_{M[p]}$ has a continuous extension to $M$; when $p \in \mathbb{Z}$, we have for any generic $p$ skeleton $M^{p},\left.u\right|_{M^{p}}$ could be approximated in $W^{1, p}\left(M^{p}, N\right)$ by a sequence of Lipschitz maps $f_{j} \in \operatorname{Lip}\left(M^{p}, N\right)$, where $f_{j}$ has a continuous extension to $M$.

In particular, one has the following

Corollary 1.4. Let $M, N$ and $p$ be the same as in Theorem 1.3. If $H_{S}^{1, p}(M, N)$ is equal to $W^{1, p}(M, N)$, then for any smooth triangulation of $M$, any map $f \in$ $C\left(M^{[p]}, N\right)$ has a continuous extension to the whole $M$.

Remark 1.1. In [HL] we show that the topological condition in the Corollary 1.4 (similarly for Theorem 1.3) is also sufficient for $H_{S}^{1, p}(M, N)$ to be equal to $W^{1, p}(M, N)$.

We note that a necessary condition for the topological condition in Corollary 1.4 to be valid is that $\pi_{[p]}(N)=0$. However $\pi_{[p]}(N)=0$ is not sufficient to guarantee that every $f \in C\left(M^{[p]}, N\right)$ has a continuous extension to the whole $M$ (see discussion in Section 3 below). This implies in particular the main theorem in [B2] has to be correctly modified.

The paper is written as follows, in Section 2 below we sketch the ideas for proving these results. Section 3 contains all the necessary counterexamples. In 
Section 4, we shall generalize these results to the case when $M$ has a nonempty boundary.

\section{Sketch of ideas}

For several results described in the introduction, parts of the proofs follow from the previous work of B. White [W1] and [W2]. As in [W1] and [W2], the homotopy extension theorem (cf. $[\mathrm{Hu}]$, p. 13) plays a crucial role in our proofs. The homotopy extension theorem says that if $A \subset B$ are nice subsets of an Euclidean space and if $H:([0,1] \times A) \cup(\{0\} \times B) \rightarrow N$ is Lipschitz, then $H$ may be extended to a Lipschitz homotopy $H:[0,1] \times B \rightarrow N$ on all of $B$. Here " $A$ is nice" means it is a Lipschitz neighborhood retractor (see $[F]$ ). Same property is true for nice pair $A \subset B$ with Lipschitz replaced by continuous. Indeed this fundamental theorem is the starting point of homotopy theory. We first give the proof of Theorem 1.1. We need the following two simple lemmas.

Lemma 2.1. Let $N$ be the same as in Theorem 1.1, $1 \leq p<n$, u be a map in $W^{1, p}\left(B_{1}^{n}, N\right)$ such that $\left.u\right|_{\partial B_{1}} \in W^{1, p}\left(\partial B_{1}, N\right)$. For $0 \leq t \leq 1$ define

$$
u_{t}(x)= \begin{cases}u(x / t), & \text { for }|x| \leq t, \\ u(x /|x|), & \text { for }|x| \geq t\end{cases}
$$

then $u_{t}$ is a continuous path in $W^{1, p}\left(B_{1}, N\right)$ with $u_{0}(x)=u(x /|x|), u_{1}=u$ and $u_{t}$ has fixed value on $\partial B_{1}$. Especially this gives us the following important boundary determination principle, for any $u, v \in W^{1, p}\left(B_{1}, N\right)$ with $\left.u\right|_{\partial B_{1}}=\left.v\right|_{\partial B_{1}} \in$ $W^{1, p}\left(\partial B_{1}, N\right)$, we may find a continuous path $w_{t}$ in $W^{1, p}\left(B_{1}, N\right)$ connecting $u$ and $v$ with $\left.w_{t}\right|_{\partial B_{1}}=\left.u\right|_{\partial B_{1}}$ for any $0 \leq t \leq 1$.

It is clear that the condition $p<n$ is essential here.

Lemma 2.2. Let $N$ be the same as in Theorem $1.1,1 \leq p<n, f_{t}$ be a continuous path in $W^{1, p}\left(\partial B_{1}^{n}, N\right), 0 \leq t \leq 1$. Define $\tilde{f}_{t}(x)=f_{t}(x /|x|)$ for $x \in B_{1}$. Then $\tilde{f}_{t}$ is a continuous path in $W^{1, p}\left(B_{1}, N\right)$.

Proof of Theorem 1.1. Suppose that $u, v \in W^{1, p}(M, N)$ and that $u$ and $v$ are $k=[p]-1$ homotopic. By choosing a generic triangulation, we may assume $\left.u\right|_{M^{k+1}},\left.v\right|_{M^{k+1}} \in W^{1, p}\left(M^{k+1}, N\right)$ and $\left.u\right|_{M^{k}},\left.v\right|_{M^{k}} \in W^{1, p}\left(M^{k}, N\right)$. Since $p \geq$ $k+1$, a simple approximation argument, Lemma 2.1 and Lemma 2.2 imply that we may assume $u$ and $v$ are Lipschitz on $M^{k+1}$ and that on each simplex of dimension higher than $k+1, u$ and $v$ are simply homogeneous degree zero extension, with respect to their barycenters, of their values on the boundary of the simplices. What these statements mean is simply that $u$ and $v$ can be connected by continuous paths in $W^{1, p}(M, N)$ to maps with described properties above. Next, since $u$ and $v$ are homotopic on $M^{k}$, by homotopy extension theorem $([\mathrm{Hu}]$, p. 13$)$ and the boundary determination principle similar to those in Lemma 2.1 and Lemma 2.2, we may further assume that $u=v$ on $M^{k}$. Again the last statement means $u$ can be connected to a map with this property by a continuous path in $W^{1, p}(M, N)$. By pushing in on each $k+1$ simplex we 
may assume for any $k+1$ simplex $\sigma,\left.u\right|_{\sigma \backslash B_{\delta}\left(x_{0}\right)}=\left.v\right|_{\sigma \backslash B_{\delta}\left(x_{0}\right)}$, here $x_{0}$ is the barycenter of $\sigma$ and $\delta$ is a tiny number. $\sigma$ is the face of several $k+2$ simplices, namely $\tau_{1}, \cdots, \tau_{r}, r \geq 2$. Now we consider a set $\Omega=\cup_{i=1}^{r} \Omega_{i}$, where $\Omega_{i} \subset \tau_{i}$ is formally equal to $\left(\overline{B_{2 \delta}\left(x_{0}\right)} \cap \sigma\right) \times[0, \varepsilon]$, for which the product we mean going in the $\tau_{i}$ in the normal direction by $\varepsilon$ length, $\varepsilon$ is another tiny number. Let $\Omega_{i}^{\prime}=\left(\overline{B_{2 \delta}\left(x_{0}\right)} \cap \sigma\right) \times\left[0, \frac{\varepsilon}{2}\right], \Omega_{i}^{\prime \prime}=\left(\overline{B_{2 \delta}\left(x_{0}\right)} \cap \sigma\right) \times\left[\frac{\varepsilon}{2}, \varepsilon\right], \Omega^{\prime}=\cup_{i=1}^{r} \Omega_{i}^{\prime}, \Omega^{\prime \prime}=$ $\cup_{i=1}^{r} \Omega_{i}^{\prime \prime}$. Then we consider a $w$ defined on $\Omega$ by setting $\left.w\right|_{\Omega^{\prime}}=v,\left.w\right|_{\partial \Omega}=\left.u\right|_{\partial \Omega}$, on each $\Omega_{i}^{\prime \prime}$ we simply do homogeneous degree zero extension. Since $p<k+2$, we have $w \in W^{1, p}(\Omega, N)$. A similar boundary determination principle as we did in Lemma 2.1 tells us $w$ can be connected to $u$ by a continuous path in $W^{1, p}(\Omega, N)$ without changing the boundary value. Let $\tilde{u}$ be defined on $M^{k+2}$ by setting $\left.\tilde{u}\right|_{\Omega}=w$ and $\left.\tilde{u}\right|_{M^{k+2} \backslash \Omega}=u$. Then $\tilde{u} \sim_{p} u$ in $W^{1, p}\left(M^{k+2}, N\right)$, and $\tilde{u}=v$ on $\sigma$. As before we may do homogeneous degree zero extension for $\tilde{u}$ on higher dimensional simplices. By this way we still call the resulting map as $u$, then we know $\left.u\right|_{\sigma}=\left.v\right|_{\sigma}$. By doing this process of deformations on all the $k+1$ simplices we may assume $\left.u\right|_{M^{k+1}}=\left.v\right|_{M^{k+1}}$. The latter fact clearly implies $u \sim_{p} v$. This proves that $u$ and $v$ being homotopic on $[p]-1$ skeleton is a sufficient condition for $u \sim_{p} v$.

Next we recall the following results proved in [W2], especially Proposition 3.3 and Theorem 3.4. Let $N$ be isometrically embedded in $\mathbb{R}^{l}$. Then given any $K>0$, there exists a $\varepsilon=\varepsilon(p, K, M, N)>0$ such that for any two maps $f, g \in W^{1, p}(M, N)$ with $|d f|_{L^{p}(M)} \leq K,|d g|_{L^{p}(M)} \leq K$ and $|f-g|_{L^{p}(M)} \leq$ $\varepsilon(p, K, M, N)$, we have $f$ and $g$ are $[p]-1$ homotopic. This plus the fact that the definition of $[p]-1$ homotopy doesn't depend on the specific choice of generic skeleton imply the necessary part of the theorem.

Proof of Proposition 1.2. By Proposition 1.1 it suffices to verify that for a triangulation of $M$, there is only one element in $C\left(M^{[p]}, N\right) / \sim_{M^{[p]-1}}$. In fact, because $k \leq[p]-1$, we know $\pi_{i}\left(M^{[p]}\right)=0$ for $1 \leq i \leq k$. Considering $\pi_{i}(N)=0$ for $k+1 \leq i \leq[p]-1$, it follows from Theorem 3 and the proof of Theorem $3^{\prime}$ in [W1] that for any $f \in C\left(M^{[p]}, N\right),\left.f\right|_{M^{[p]-1}}$ is homotopic to a constant map.

Proof of Theorem 1.2. Suppose $u \in W^{1, p}(M, N)$ can be connected to a smooth map $v \in C^{\infty}(M, N)$, from Theorem 1.1 we know $u$ and $v$ are $[p]-1$ homotopic. Hence for any generic $[p]-1$ skeleton $M^{[p]-1}$ we have $\left.u\right|_{M^{[p]-1}}$ is homotopic to

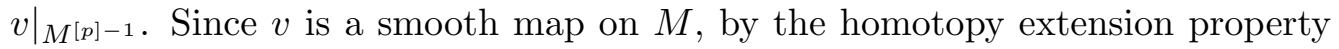
we may find a continuous extension of $\left.u\right|_{M^{[p]-1}}$ to $M$ (see p. 13 of [Hu]).

On the other hand, if there exists a generic $[p]-1$ skeleton $M^{[p]-1}$ such that $\left.u\right|_{M[p]-1}$ has a continuous extension $w \in C(M, N)$, then we may pick up a $v \in C^{\infty}(M, N)$ such that $v$ is homotopic to $w$. Clearly $v$ is $[p]-1$ homotopic to $u$, and hence $v \sim_{p} u$ by Theorem 1.1 .

If for $u \in W^{1, p}(M, N)$ we may find $u_{j} \in C^{\infty}(M, N)$ such that $u_{j} \rightarrow u$ in $W^{1, p}(M, N)$, then it follows from Proposition 3.3 and Theorem 3.4 in [W2] that $u_{j}$ is $[p]-1$ homotopic to $u$ for $j$ large (one may also refer to the proof of 
Theorem 1.1). For a generic skeleton $M^{[p]-1},\left.u\right|_{M^{[p]-1}}$ is homotopic to $\left.u_{j}\right|_{M^{[p]-1}-1}$, hence it follows from homotopy extension property that $\left.u\right|_{M[p]-1}$ has a continuous extension to $M$.

Proof of Theorem 1.3. Let $u$ be a map in $H_{S}^{1, p}(M, N)$, then there exists a sequence of smooth maps, namely $u_{j} \in C^{\infty}(M, N)$, such that $u_{j} \rightarrow u$ in $W^{1, p}(M, N)$. Hence for generic skeleton $M^{[p]},\left.\left.u_{j}\right|_{M^{[p]}} \rightarrow u\right|_{M^{[p]}}$ in $W^{1, p}\left(M^{[p]}, N\right)$. If $p \notin \mathbb{Z}$, by Sobolev embedding theorem we know $u \in$ $C\left(M^{[p]}, N\right)$ and $\left.\left.u_{j}\right|_{M[p]} \sim u\right|_{M[p]}$. Now it follows from homotopy extension property that $\left.u\right|_{M^{[p]}}$ has a continuous extension. If $p \in \mathbb{Z}$, we just take $\left.u_{j}\right|_{M^{p}}$ as $f_{j}$.

\section{Counterexamples}

From Corollary 1.2 we see that when $1 \leq p<\operatorname{dim} M$, a necessary condition for $H_{W}^{1, p}(M, N)=W^{1, p}(M, N)$ is that for any continuous map $f: M^{[p]} \rightarrow N$ one may find a continuous extension of $\left.f\right|_{M^{[p]-1}}: M^{[p]-1} \rightarrow N$ to a map in $C(M, N)$. This condition is also necessary and sufficient for every map in $W^{1, p}(M, N)$ to be connected by a continuous path in $W^{1, p}(M, N)$ to some smooth maps. One realizes at once that there are obstructions for such a topological statement to be true. Indeed one may find many elaborate examples by the so called obstruction theory in classical homotopy theory (see [Wh]). Here we shall deduce the existence of such examples by the following

Proposition 3.1. Let $X$ and $Y$ be two topological spaces, $k \geq 1$ be a fixed integer. Assume $X$ has two $C W$ complex structures, namely $\left(X^{j}\right)_{0 \leq j<\infty}$ and $\left(\tilde{X}^{j}\right)_{0 \leq j<\infty}$. If for any $f \in C\left(X^{k}, Y\right),\left.f\right|_{X^{k-1}}$ has a continuous extension to $X$, then for any $\tilde{f} \in C\left(\tilde{X}^{k}, Y\right),\left.\tilde{f}\right|_{\tilde{X}^{k-1}}$ has a continuous extension to $X$.

This statement may be well-known in topology though we are not aware where one can find a proof. We thank Sylvain Cappell for showing us a simple proof by the cellular approximation theorem (cf. [Wh], p. 77). By Proposition 3.1, we see the condition for any smooth triangulation of $M$, any $f \in C\left(M^{[p]}, N\right)$, $\left.f\right|_{M^{[p]-1}}$ has a continuous extension to $M$ is equivalent to say for a given CW complex structure $\left(M^{i}\right)_{0 \leq i \leq \operatorname{dim} M}$ of $M$, any map $f \in C\left(M^{[p]}, N\right),\left.f\right|_{M^{[p]-1}}$ can be extended to a map in $C(M, N)$. Though smooth triangulations are favorable for analysis, it is much easier to do homotopy theory with $\mathrm{CW}$ complex structures. Now we give an example (due to S. Cappell and F. Bogomolov) which says the latter cannot always be done. We start with the natural CW complex structure of $\mathbb{C P}^{n}, \mathbb{C P}^{0} \subset \mathbb{C P}^{1} \subset \cdots \subset \mathbb{C P}^{n}$ with the obvious attaching maps. By the standard fibration $\mathbb{C P}^{n}=\mathbb{S}^{2 n+1} / \mathbb{S}^{1}$ we see $\pi_{i}\left(\mathbb{C P}^{n}\right)=0$ for all $0 \leq i \leq 2 n-1$ and $i \neq 2$. Now given any $m>n \geq 1$, we note the identity map $i: \mathbb{C P}^{1} \subset \mathbb{C P}^{m} \rightarrow \mathbb{C P}^{1} \subset \mathbb{C P}^{n}$ cannot be extended continuously on the whole $\mathbb{C P}^{m}$. Indeed, suppose it has a continuous extension $g$, let $\alpha, \beta$ be the 
cohomology class in $H^{2}\left(\mathbb{C P}^{m}\right)$ and $H^{2}\left(\mathbb{C P}^{n}\right)$ corresponding to $\mathbb{C P}^{1}$ respectively. These are generators of the cohomology rings $H^{*}\left(\mathbb{C P}^{m}\right)$ and $H^{*}\left(\mathbb{C P}^{n}\right)$ which are isomorphic to $\mathbb{Z}[\alpha] /\left\{\alpha^{m+1}=0\right\}$ and to $\mathbb{Z}[\beta] /\left\{\beta^{n+1}=0\right\}$ respectively. Clearly $g^{*}(\beta)=\alpha$, hence $\alpha^{n+1}=g^{*}\left(\beta^{n+1}\right)=0$, this contradicts to the fact that $m>n$. In particular, one may take $M=\mathbb{C P}^{2}, N=\mathbb{C P}^{1}$ and $p=3$. In the natural CW complex structure of $\mathbb{C P}^{2}, M^{2}=M^{3}=\mathbb{C P}^{1}$. Then for the above map $i: M^{3}=\mathbb{C P}^{1} \rightarrow N,\left.i\right|_{M^{2}}$ has no continuous extension to the whole $\mathbb{C P}^{2}$. This implies there is a map in $W^{1,3}\left(\mathbb{C P}^{2}, \mathbb{C P}^{1}\right)$ which is not in $H_{W}^{1,3}\left(\mathbb{C P}^{2}, \mathbb{C P}^{1}\right)$ and which can not be connected to a smooth map by a continuous path in $W^{1,3}\left(\mathbb{C P}^{2}, \mathbb{C P}^{1}\right)$. If we consider also $W^{1,3}\left(\mathbb{C P}^{3}, \mathbb{C P}^{2}\right)$ and $W^{1,2}\left(\mathbb{S}^{3}, \mathbb{S}^{2}\right)$, we note that $\pi_{[p]}(N)=0$ is neither necessary nor sufficient to guarantee the topological condition in Corollary 1.2. Similar to Proposition 3.1, we have

Proposition 3.2. Let $X$ be a topological space with two $C W$ complex structures, namely $\left(X^{i}\right)_{0 \leq i<\infty}$ and $\left(\tilde{X}^{i}\right)_{0 \leq i<\infty}, k$ be a fixed nonnegative integer, and $Y$ be a connected topological space with $\pi_{k}(Y)=0$. If every $f \in C\left(X^{k}, Y\right)$ has a con-

tinuous extension to $X$, then every $\tilde{f} \in C\left(\tilde{X}^{k}, Y\right)$ has a continuous extension to $X$ too.

By Corollary 1.4 and Proposition 3.2, we may easily find a counterexample to Theorem 1 of [B2], which says $H_{S}^{1, p}(M, N)=W^{1, p}(M, N)$ if and only if $\pi_{[p]}(N)=0$. Indeed, if we take $M=\mathbb{C P}^{3}, N=\mathbb{C P}^{2}$ and $p=3$, then $\pi_{3}(N)=0$. Since $i: M^{3}=\mathbb{C P}^{1} \subset M \rightarrow \mathbb{C P}^{1} \subset N$ has no continuous extension as above, one concludes by Proposition 3.2 that there is a continuous (hence we may also assume Lipschitz) map from a three dimension skeleton of a smooth triangulation of $\mathbb{C P}^{3}=M$ which cannot be extended to a map in $C(M, N)$. The latter would imply there is a map in $W^{1,3}(M, N)$ which is not in $H_{S}^{1,3}(M, N)$.

\section{Generalizations to the Case $\partial M \neq \emptyset$}

Essentially all the results described in the introduction have their analogies in the case $\partial M \neq \emptyset$. Throughout this section we shall assume $M$ is a connected smooth compact Riemannian manifold with a nonempty boundary, and $N$ is a connected smooth compact Riemannian manifold without boundary. Denote $n=\operatorname{dim} M$.

Similar to Theorem 1.1, one can easily show for any $1 \leq p<n$, any two maps $u, v \in W^{1, p}(M, N)$,

$$
u \sim_{p} v \Longleftrightarrow u \text { and } v \text { are }[p]-1 \text { homotopic. }
$$

The result corresponding to Corollary 1.1 is

Corollary 4.1. If $1 \leq p<n$ and $\pi_{i}(N)=0$ for $[p] \leq i \leq n-1$, then the two maps below are bijections,

$$
C(M, N) / \sim_{M} \longleftarrow \operatorname{Lip}(M, N) / \sim_{M, L i p} \longrightarrow W^{1, p}(M, N) / \sim_{p} .
$$


We should note that it is not required in Corollary 4.1 that $\pi_{n}(N)=0$. This is simply because when we do the last step of extension, we may push the topological obstructions out through the boundary. Corresponding to Corollary 1.2 we have

Theorem 4.1. If $1 \leq p<n$, then the following three assertions are equivalent

- Every map in $W^{1, p}(M, N)$ can be connected to a smooth map by a continuous path in $W^{1, p}(M, N)$;

- For any triangulation of $M$, any $f \in C\left(M^{[p]}, N\right),\left.f\right|_{M^{[p]-1}}$ has a continuous extension to $M$;

- For any triangulation of $M$, any $f \in C\left(M^{[p]}, N\right),\left.f\right|_{M[p]-1}$ has a continuous extension to $M^{n-1}$.

The latter two conditions are also necessary for $H_{W}^{1, p}(M, N)$ to be equal to $W^{1, p}(M, N)$.

Theorem 4.1 has the following corollary.

Corollary 4.2. Let $1 \leq p<n$. If either $[p]=1$ or $[p] \geq 2$ and $\pi_{i}(N)=0$ for $[p] \leq i \leq n-2$, then any map in $W^{1, p}(M, N)$ can be connected in $W^{1, p}(M, N)$ to a smooth map.

We note Corollary 4.2 covers Theorem 4 in [BL]. Finally we have similar results for strong density of smooth maps.

Theorem 4.2. Let $1 \leq p<n$. If $H_{S}^{1, p}(M, N)=W^{1, p}(M, N)$, then for any smooth triangulation of $M$, any $f \in C\left(M^{[p]}, N\right)$ has a continuous extension to the whole $M$.

We refer the readers to our paper [HL] for the detailed expositions and proofs.

\section{Acknowledgments}

The authors wish to thank Sylvain Cappell and Fedor Bogomolov for some valuable suggestions. The research of the first author is supported by a Dean's Dissertation Fellowship. The research of the second author is supported by a NSF grant.

\section{References}

[B1] F. Bethuel, A characterization of maps in $H^{1}\left(\mathbb{B}^{3}, \mathbb{S}^{2}\right)$ which can be approximated by smooth maps, Ann. Inst. H. Poincar Anal. Non Linaire 7 (1990), 269-286.

[B2] The approximation problem for Sobolev maps between two manifolds, Acta Math. 167 (1991), 153-206.

[BBC] F. Bethuel, H. Brezis, and J. M. Coron, Relaxed energies for harmonic maps, Variational Methods, Birkhäuser, Boston, 1990, pp. 37-52.

[BCDH] F. Bethuel, J. M. Coron, F. Demengel and F. Helein, A cohomological criterion for density of smooth maps in Sobolev spaces between two manifolds, Nematics. Ed. J. M. Coron et. al., NATO Adv. Sci. Inst. Ser. C Math. Phys. Sci. 332, Kluwer Academic Publishers, 1990, pp. 15-23. 
[BL] H. Brezis and Y. Y. Li, Topology and Sobolev spaces, C. R. Acad. Sci. Paris Sr. I Math 331 (2000), 365-370.

[Bu] F. E. Burstall, Harmonic maps of finite energy from noncompact manifolds, J. London Math. Soc. 30 (1984), 361-370.

[F] H. Federer, Geometric measure theory, Die Grundlehren der mathematischen Wissenschaften, Band 153, Springer-Verlag, New York 1969.

[H] P. Hajlasz, Approximation of Sobolev mappings, Nonlinear Anal. 22 (1994), 15791591.

[HL] F. B. Hang and F. H. Lin, Topology of Sobolev mappings, II, preprint.

[HR] R. Hardt and T. Rivière, preprint (2000).

[Hu] S. T. Hu, Homotopy Theory, Academic Press, New York, 1959.

[M] J. R. Munkres, Elementary differential topology, Princeton University Press, Princeton, New Jersey, 1963.

[PR] M. R. Pakzad and T. Rivière, Weak density of smooth maps for the Dirichlet energy between manifolds, preprint (2000).

[SU] R. Schoen and K. Uhlenbeck, Approximation theorems for Sobolev mappings, preprint (1984).

[SY] R. Schoen and S. T. Yau, The existence of incompressible minimal surfaces and topology of three dimensional manifolds with non-negative scalar curvature, Ann. of Math. 110 (1979), 127-142.

[W1] B. White, Infima of energy functions in homotopy classes of mappings. J. Differential Geom. 23 (1986), 127-142.

[W2] Homotopy classes in Sobolev spaces and the existence of energy minimizing maps, Acta Math. 160 (1988), 1-17.

[Wh] G. W. Whitehead, Elements of homotopy theory, Graduate Texts in Mathematics 61, Springer-Verlag, New York, 1978.

Courant Institute, 251 Mercer Street, New York, NY 10012.

E-mail address: fengbo@cims.nyu.edu

E-mail address: linf@cims.nyu.edu 